\begin{tabular}{ll}
\hline \hline MINING AND METALLURGY INSTITUTE BOR & ISSN: 2334-8836 \\
& UDK: 622 \\
\hline \hline
\end{tabular}

\title{
SELECTION OF DRILLING AND BLASTING OPERATIONS AT THE OPEN PIT "POTRLICA" PLJEVLJA
}

\begin{abstract}
Considering that in obtaining the mineral resources about $70 \%$ of mining operations belongs to drilling and blasting, where there are the highest costs, this paper is aimed to description and proof the modern way of mining that imply maximum effects both in technological and in terms of safety.

Keywords: technology, mining, maximum effects, safety
\end{abstract}

\section{INTRODUCTION}

The Coal Basin Pljevlja, according to its economic importance, is right behind the Maoče Basin. Geomorphologically viewed, it almost entirely covers the Pljevlja valley, located in the middle course of the river Ćehotina in a place where the left tributary Vezišnica flows into it.

The area of Pljevlja Basin with the neogene sediments under the lake is $18 \mathrm{~km}^{2}$, while the Pljevlje Basin in a narrow sense covers an area of about $12 \mathrm{~km}^{2}$ and is bounded by the points whose coordinates are [1]:

$\mathrm{X}$ from 4798100 to 4801530

Y from 6607000 to 6612400

\section{DRILLING AND BLASTING OPERATIONS}

Drilling and blasting operations are defined for exploitation of overburden in the amount of 4,000,000 $\mathrm{m}^{3} \mathrm{c} \mathrm{m}$ of the waste and $1,500,000$ tons of coal.

Considering the applied discontinuous system of exploitation, the requirements of satisfactory coal granulation for consumer goods, the blasting is carried on agitation, it is rowed and millisecond.

Drilling and blasting of coal and overburden, defined according to the bench heights, is carried out at 10 and 15 meters. Powdered and water plastic explosives are used for blasting

\subsection{Drilling of boreholes}

At the open pit "Potrlica", drilling of boreholes of diameter $115 \mathrm{~mm}$ is performed. The boreholes are angled with the slope angle to the horizontal of $\alpha=75^{\circ}$ on coal and overburden. The boreholes are drilled parallel to the bench slope. Length of boreholes varies, depending on height of benches that is thickness of overburden and coal seam thickness.

Taking into account all listed properties of working environment at the open pit "Potrlica", the rotary drilling system was applied.

\footnotetext{
* Faculty of Technical Sciences, Kosovska Mitrovica
} 
For this purpose, the hydraulic rotary drills are used. These are [2]:

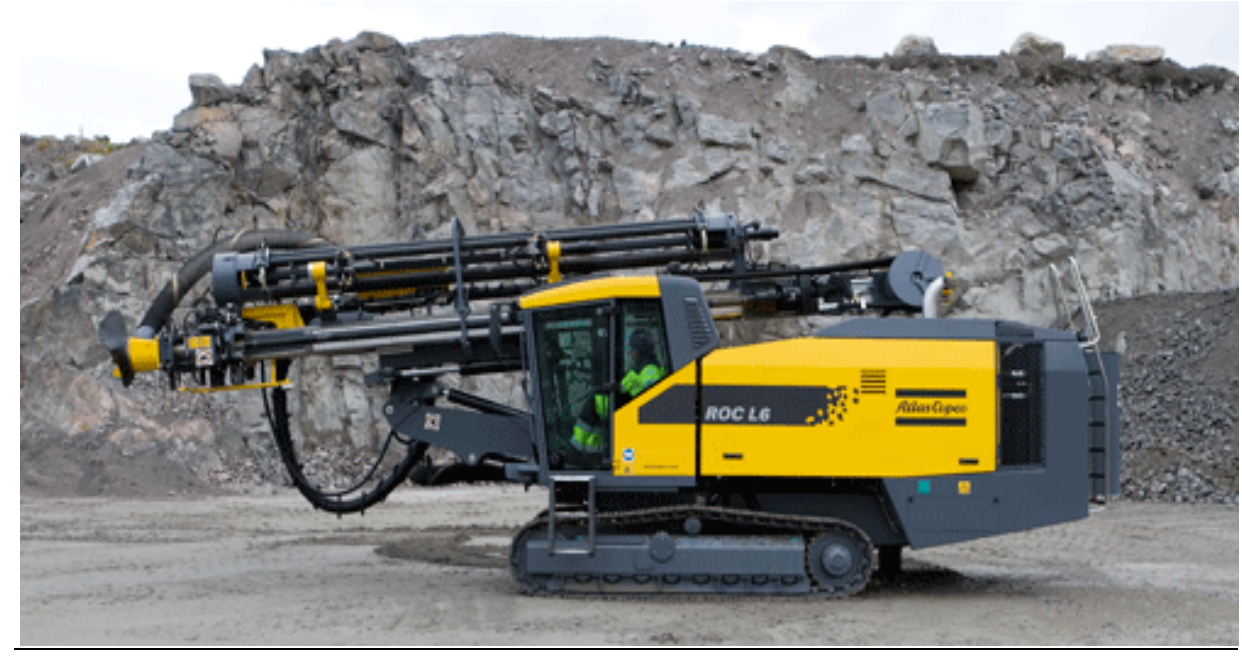

Figure 1 ATLAS COPCO ROC L6-25

\subsection{Drilling and blasting operations on} overburden

Blasting parameters on overburden using the explosive, type AMONEX

Calculation of explosive specific consumption

Calculation will be made for explosive AMONEX-3.

Calculation of specific consumption of explosive was made using the Lares formula and it is:

$$
\begin{aligned}
q & =\frac{q_{1} x \text { sxvxexd }}{\Delta}= \\
& =\frac{0, .091 \times 1 \times 1 \times 1,3 \times 1}{1.05}=0.11 \mathrm{~kg} / \mathrm{m}^{3}
\end{aligned}
$$

Calculation of drilling length and borehole pitting

$$
\text { For } \mathrm{H}=10 \mathrm{~m}
$$

$$
L=\frac{H}{\sin \alpha}+l_{p r}=\frac{10}{\sin 75^{0}}+1=11.35 m
$$

Network of boreholes and rapprochement coefficient of boreholes

Drilling of boreholes should be done in a triangular arrangement in two or three rows. The coefficient of bringing together should be equal to $\mathrm{m}=1 ; \mathrm{m}=\mathrm{a} / \mathrm{W}$. 

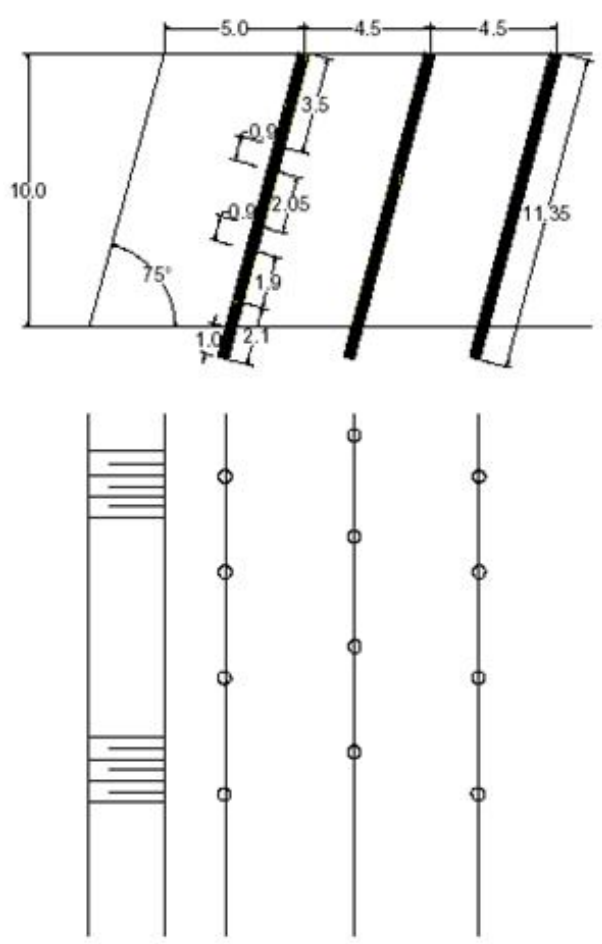

Figure 2 Construction of blast filling of borehole on overburden for $\mathrm{H}=10 \mathrm{~m}$ - schematic view

\section{Calculation the least resistance line}

Will be carried out according to the model of S. Davidov

$$
\begin{aligned}
W & =53 x k_{t} x d x \sqrt{\frac{\Delta}{\gamma_{s}}}= \\
& =53 x 1,1 x 0.115 x \sqrt{\frac{1,03}{1,9}}=4.93 \approx 5 \mathrm{~m}
\end{aligned}
$$

\subsection{Calculation of distance between} boreholes

$$
\begin{aligned}
& \text { For } \mathrm{H}=10, \\
& \quad a=m \times W=1 \times 5=5 \mathrm{~m},
\end{aligned}
$$

adopted as $5 \mathrm{~m}$.

\subsection{Calculation of distance between the rows of boreholes}

For $\mathrm{H}=10$, $b=0.87 \times W=0.87 \times 5=4.35 \approx 4.5 \mathrm{~m}$, adopted as $4.5 \mathrm{~m}$.

2.5 Calculation the length of stemming

For bench height $\mathrm{H}=10 \mathrm{~m}$

$L_{s}=(0.75 \div 1.0) x \mathrm{~W}=3.75 \div 5 \mathrm{~m}$

$L_{s}=(20 \div 40) x d=2.3 \div 4.6 \mathrm{~m}$

The adopted length of STEMMING is $1_{\mathrm{s}}=3.5 \mathrm{~m}$.

\subsection{Calculation the explosive quantity} in one borehole

For calculation of filling in borehole, it is started from the basic form [3]

$$
Q=q x V(k g)
$$




\section{CONCLUSION}

It is necessary for exploitation to comply fully with parameters (amount of explosives, deceleration intervals, minefield schemes) as given in the design solution. Also, if for any reason there is a change of explosives or other blasting parameters, they should be thoroughly checked and subsequently analyzed.

During exploitation, it is necessary to carry out a continuous monitoring of blasting results (quality of fragmentation, the impact of mining on surrounding objects, etc.) for feedback analysis and possible correction of parameters that were unknown or did not take into account in design.

\section{REFERENCES}

[1] Additional Mining Design of the Open Pit "Potrlica“" Pljevlja, Coal Mine Pljevlja, 2008 (in Serbian);

[2] N. Popović Machines and Devices in Exploitation of Mineral Deposits, (in Serbian);

[3] V. Pavlović, Technology of Open Pit Mining, 1992 (in Serbian). 


\begin{tabular}{ll}
\hline \hline INSTITUT ZA RUDARSTVO I METALURGIJU BOR & ISSN: 2334-8836 \\
& UDK: 622 \\
\hline \hline
\end{tabular}

\section{IZBOR BUŠAČKO-MINERSKIH RADOVA NA POVRŠINSKOM KOPU „POTRLICA“ PLJEVLJA}

Izvod

Obzirom da se pri dobijanju mineralnih sirovina od rudarskih operacija oko $70 \%$ pripada bušenju i miniranju gde su i najveći troškovi, ovim radom želimo da opišemo i dokažemo savremeni način miniranja koji podrazumevaju maksimalne efekte kako u tehnološkom tako i u sigurnosnom smislu.

Ključne reči: tehnologija, miniranje, maksimalni efekti, sigurnost

\section{UVOD}

Pljevaljski ugljonosni basen po svom ekonomskom značaju, nalazi se odmah iza maočkog basena. Geomorfološki posmatrano on skoro $\mathrm{u}$ potpunosti obuhvata pljevaljsku kotlinu, koja se nalazi u središnjem toku reke Ćehotine, na mestu gde se u nju uliva leva pritoka Vezišnica.

Površina pljevaljske kotline pod jezerskim neogenim sedimentima iznosi $18 \mathrm{~km}^{2}$ dok pljevaljski basen u užem smislu obuhvata površinu od oko $12 \mathrm{~km}^{2}$, a omeđena je tačkama čije su coordinate [1]:

$\mathrm{X}$ od 4798100 do 4801530

Y od 6607000 do 6612400

\section{BUŠAČKO MINERSKI RADOVI}

Izvođenje bušačko-minerskih radova definisani su za eksploataciju otkrivke u količini od $4.000 .000 \mathrm{~m}^{3}$ čm otkrivke i $1.500 .000 \mathrm{t}$ uglja.
Obzirom na primenjeni diskontinualni sistem eksploatacije, potrebe zadovoljavajuće granulacije uglja za široku potrošnju, miniranje se izvodi na rastresanje, višeredno je i milisekundno.

Bušenje i miniranje na uglju i otkrivci, saglasno definisanim visinama etaža, vrši se na 10 i $15 \mathrm{~m}$. Za miniranje koriste se praškasti i vodoplastični eksplozivi.

\subsection{Bušenje minskih bušotina}

Na P. K. „Potrlica" vrši se bušenje minskih bušotina prečnika $115 \mathrm{~mm}$. Bušotine su kose sa uglom nagiba prema horizontali od $\alpha=75^{0}$ na uglju i otkrivci. Bušotine se buše paralelno kosini etaže. Dužina bušotina je različita, a u zavisnosti od visine etaže, odnosno moćnosti otkrivke i debljine ugljenog sloja.

Uzimajući u obzir sva nabrojana svojstva radne sredine na P. K. „Potrlica", primjenjen je rotacioni sistem bušenja.

\footnotetext{
* Fakultet Tehničkih Nauka, Kosovska Mitrovica
} 
Za ovu svrhu koriste se hidraulične rotacione bušilice [2]. To su:
BÖHLERR TCD-222

$1 \mathrm{kom}$.

ATLAS COPCO ROC L $\mathrm{L}_{6}-251 \mathrm{kom}$.

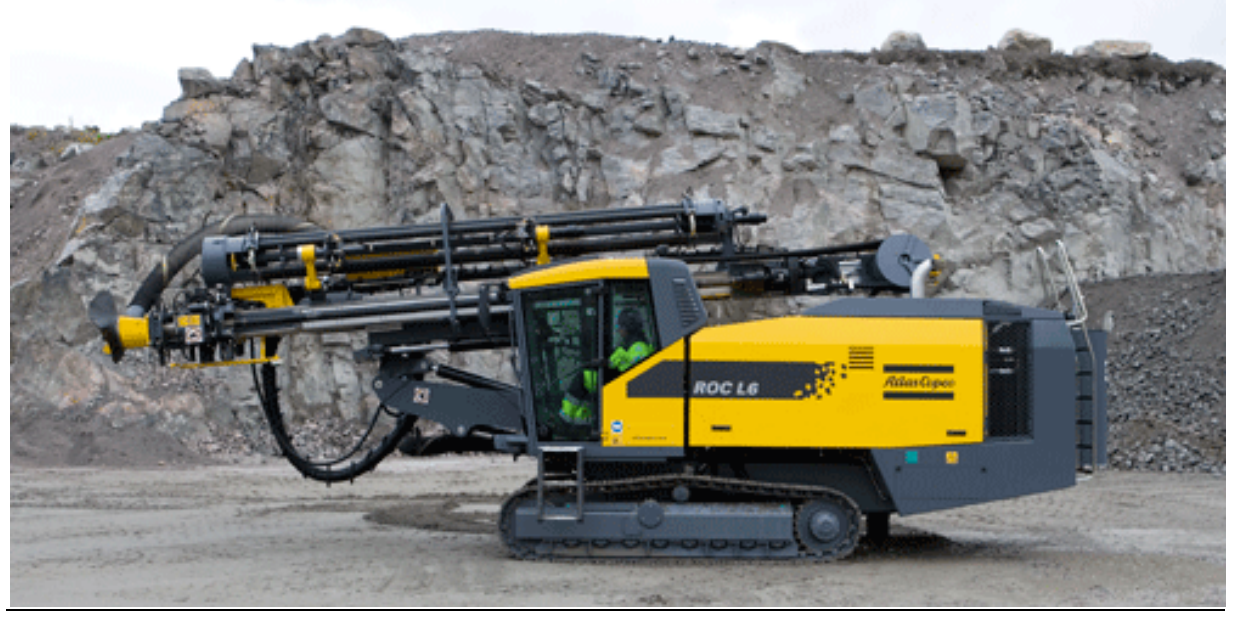

SI. 1. Bušilica ATLAS COPCO ROC L6-25

\subsection{Bušačko minerski radovi} na otkrivci

Parametri miniranja na otkrivci primenom eksploziva tipa Amonex

Proračun specifične potrošnje eksploziva

Proračun će biti izvršen za eksplozivom Amonex-3.

Proračun specifične potrošnje eksploziva izvršen je primenom Laresove formule i iznosi:

$$
\begin{aligned}
q & =\frac{q_{1} \cdot s \cdot v \cdot e \cdot d}{\Delta}=\frac{0,091 \cdot 1 \cdot 1 \cdot 1,3 \cdot 1}{1,05}= \\
& =0,11 \mathrm{~kg} / \mathrm{m}^{3}
\end{aligned}
$$

Proračun dužine bušenje i probušenja bušotina

$\mathrm{Za} \mathrm{H}=10 \mathrm{~m}$

$$
\begin{aligned}
L & =\frac{H}{\sin \alpha}+l_{p r}=\frac{10}{\sin 75^{0}}+1= \\
& =11,35 \mathrm{~m}
\end{aligned}
$$

Mreža minskih bušotina i koeficijent zbliženja bušotina

Bušenje minskih bušotina treba vršiti u trougaonom rasporedu i to $\mathrm{u}$ dva ili tri reda. Koeficijent zbliženja treba da iznosi $\mathrm{m}=1 ; \mathrm{m}=\mathrm{a} / \mathrm{W}$. 

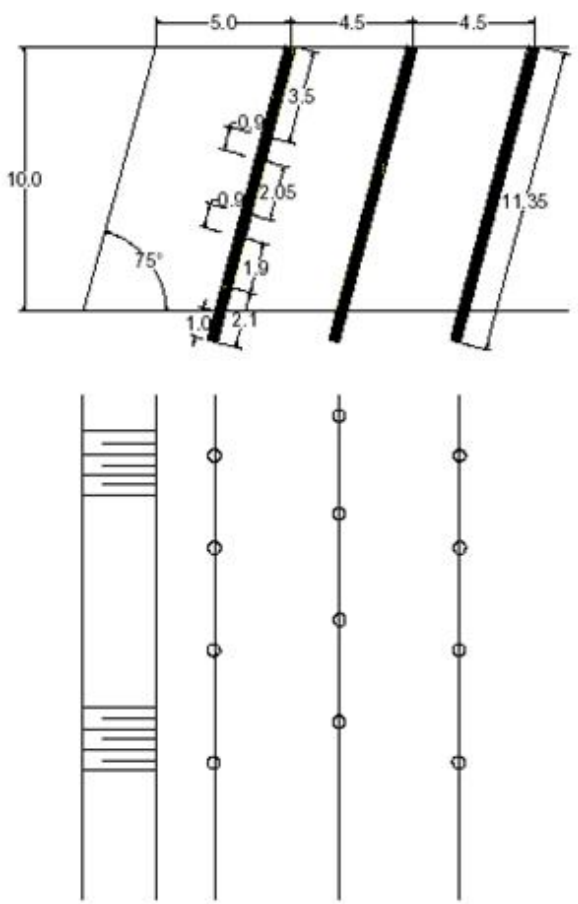

SI. 2. Konstrukcija minskog punjenja bušotine na otkrivci za $\mathrm{H}=10 \mathrm{~m}$ - ̌̌ematski prikaz.

\section{Proračun linije najmanjeg otpora}

Izvršiće se prema obrascu S. Davidova

$$
\begin{aligned}
W & =53 \cdot k_{t} \cdot d \cdot \sqrt{\frac{\Delta}{\gamma_{s}}}= \\
& =53 \cdot 1,1 \cdot 0,115 \cdot \sqrt{\frac{1,03}{1,9}}=4,93 \approx 5 m
\end{aligned}
$$

\subsection{Proračun rastojanja između} bušotina

$\mathrm{Za} \mathrm{H}=10 \mathrm{~m}$,

$a=m \times W=1 \times 5=5 m$,

usvaja se $5 \mathrm{~m}$.

2.4. Proračun rastojanja između redova bušotina

$\mathrm{Za} \mathrm{H}=10 \mathrm{~m}$, $\mathrm{b}=0,87 \times \mathrm{W}=0,87 \times 5=4,35 \approx 4,5 \mathrm{~m}$, usvaja se $4,5 \mathrm{~m}$.

2.5. Proračun dužine minskog čepa

$\mathrm{Za}$ visinu etaže $\mathrm{H}=10 \mathrm{~m}$

$l_{\check{c}}=(0,75 \div 1,0) \times W=3,75 \div 5 \mathrm{~m}$

$l_{\check{c}}=(20 \div 40) \times d=2,3 \div 4,6 m$

Usvojena dužina minskog čepa iznosi $1_{\check{c}}=3,5 \mathrm{~m}$.

\subsection{Proračun količine eksploziva u} jednoj bušotni

Za proračun punjenja u minskoj bušotini polazi se od osnovnog obrasca [3]

$$
Q=q \times V(k g)
$$




\section{ZAKLJUČAK}

Neophodno je da se pri eksploataciji u potpunosti ispoštuju parametri (količine eksploziva, intervali usporenja, šeme minskog polja) koji su dati u projektnom rešenju. Takođe, ukoliko iz bilo kojih razloga dođe do promene eksploziva ili nekog drugog parametra miniranja, iste treba detaljno proveriti i naknadno analizirati.

Pri eksploataciji je neophodno vršiti stalno praćenje rezultata miniranja (kvalitet usitnjavanja, uticaj miniranja na okolne objekte i dr.) radi povratne analize i eventualne korekcije parametara koji nisu bili poznati ili uzeti u obzir prilikom projektovanja.

\section{LITERATURA}

[1] Dopunski rudarski projekat PK „Potrlica“ Pljevlja, Rudnik uglja A.D. Pljevlja, 2008

[2] N. Popović, Mašine i uređaji u eksploataciji LMS

[3] V. Pavlović, Tehnologija površinskog otkopavanja, 1992 